\title{
Norois
}

Environnement, aménagement, société

244 | 2017

Sport, immigration, urbanisation littorale, climat sahélien, biodiversité

\section{Abdelkader MoHAINE, La Géographie et l'Aménagement au Maroc, Regards croisés}

Mustapha El Hannani

\section{OpenEdition}

Journals

Édition électronique

URL : http://journals.openedition.org/norois/6181

DOI : $10.4000 /$ norois. 6181

ISBN : 978-2-7535-7420-5

ISSN : $1760-8546$

Éditeur

Presses universitaires de Rennes

Édition imprimée

Date de publication : 30 décembre 2017

Pagination : 91-92

ISBN : 978-2-7535-7418-2

ISSN : 0029-182X

\section{Référence électronique}

Mustapha El Hannani, «Abdelkader Mohaine, La Géographie et l'Aménagement au Maroc, Regards

croisés », Norois [En ligne], 244 | 2017, mis en ligne le 30 décembre 2017, consulté le 04 janvier 2021.

URL : http://journals.openedition.org/norois/6181; DOI : https://doi.org/10.4000/norois.6181

\section{(c) Tous droits réservés}




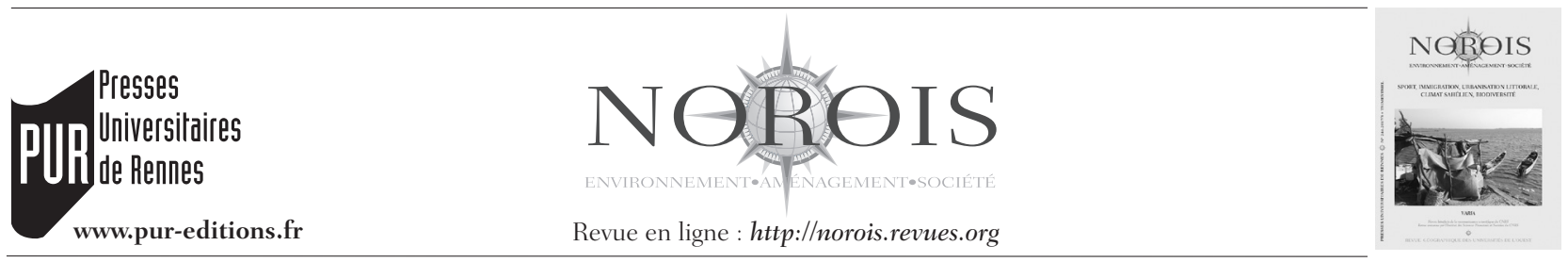

\section{COMPTES RENDUS BIBLIOGRAPHiQueS}

\section{Abdelkader Mohaine, 2017, La Géographie et l'Aménagement au Maroc, Regards croisés, Casablanca, de Éd. Afrique-Orient.}

Cet ouvrage, à la fois conceptuel et historique, est destiné à un public d'étudiants et d'enseignantschercheurs en géographie et dans une certaine mesure aux professionnels de l'aménagement des territoires. En effet, il permet de comprendre la généalogie de plusieurs notions et concepts aussi complexes que l'aménagement, le territoire ou l'espace, indispensables dans la construction d'une culture d'aménagement pour le futur aménageur marocain, et surtout le géographe.

Cet ouvrage est organisé en deux chapitres. Le premier est une synthèse retraçant le parcours théorique et historique du concept d'aménagement, sujet dont le contenu relève de plusieurs disciplines : géographie, droit et histoire, urbanisme...

L'aménagement est à la fois une notion, un concept et un domaine faisant appel à diverses compétences et savoirs. L'auteur appuie son exposé en faisant référence à de nombreux auteurs. L'aménagement est également indissociable de deux autres notions fondamentales qui sont celles d'espace et de territoire. Ce sont ces deux dimensions qui donnent plus de légitimité à la géographie comme science de l'aménagement du territoire. Cette légitimité résulte aussi du fait qu'il s'agit d'une discipline de synthèse entre éléments physiques et humains et mobilisant des notions appropriées à l'espace comme l'échelle.

Dans son deuxième chapitre consacré plus spécifiquement à la question de l'aménagement du territoire au Maroc, l'auteur, en s'appuyant sur sa

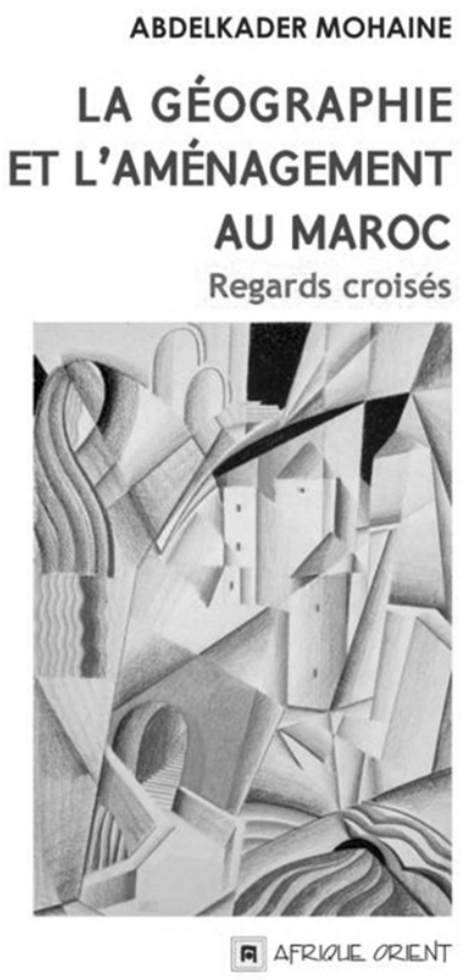

propre expérience d'enseignant-chercheur et de pratiquant (participation à la mise en place de plans d'aménagement) tente de retracer en trois temps ou périodes, le parcours de ce concept étranger à la culture de l'État marocain.

Le premier temps correspondant à la période post-coloniale jusqu'au début des années 1990 
(période la plus longue) est qualifié de «néant» dans le domaine de l'aménagement du territoire. Il est difficile de parler de politique d'aménagement au sens propre du terme pendant cette période où plusieurs plans ont bien été élaborés et actions menées concernant quelques domaines comme l'agriculture, mais sans réels succès.

Cependant, deux grandes actions caractérisent cette longue période : la politique de la grande hydraulique et le début de l'instauration de la régionalisation version marocaine, avec un élément spécifique qui est que le découpage régional est assujetti à des obsessions d'ordre sécuritaire. C'est le point commun caractéristique que partagent tous les découpages territoriaux au Maroc. Sur le plan économique, les plans quinquennaux n'ont jamais atteint leurs objectifs, en partie car le programme d'ajustement structurel imposé par le FMI imposera à l'État de revoir ses objectifs et se traduira par un recul de son rôle, surtout dans les domaines sociaux.

Il faudra attendre les années 2000 et l'arrivée d'un nouveau souverain au pouvoir pour enfin voir une lente émergence d'une approche territoriale des problèmes que connaît un pays en pleine transformation socio-spatiale. En effet, face aux enjeux d'une population très jeune et une forte croissance urbaine en relation avec un exode rural toujours soutenu, le pouvoir central donnera un coup d'accélérateur et mettra en place plusieurs outils (Schéma National d'Aménagement du Territoire (SNAT), schéma d'aménagement régional (SRAT, code d'urbanisme...) qui s'inscrivent pleinement dans une réelle approche d'aménagement du territoire.

Parallèlement à cette évolution, de nouveaux/ anciens concepts s'imposent comme le développement local, permettant une réelle implication de la société civile dans des projets de développement.
C'est dans ce contexte que les instances concernées par l'aménagement du territoire connaissent une réorganisation et une redéfinition de leurs missions.

L'auteur termine l'analyse de ce long processus de « construction » d'une politique d'aménagement du territoire par un constat sous forme de regret ou d'amertume; la politique d'aménagement du territoire au Maroc reste calquée sur le modèle français.

L'auteur énumère toutes les structures administratives qui ont vu le jour avec la mise en place d'une politique d'aménagement associée à un nouveau découpage territorial. Cependant, il est toujours prématuré de parler d'actions concrètes résultant de cette nouvelle politique. L'action territoriale de l'État prend souvent la forme de grands programmes (Maroc vert, plan azur, agences de développement...) qui sont loin de répondre aux enjeux structurants la problématique du développement territorial au Maroc comme les relations villes-campagnes, l'articulation plaine-montagne et la littoralisation croissante. Mais le plus important pour l'auteur, c'est le défi majeur du capital humain, c'est-à-dire la formation du futur citoyen.

Le Pr. A. Mohaine s'interroge enfin sur la place de la géographie et du géographe marocain dans ce contexte et face à cette problématique de l'aménagement du territoire au Maroc. Pour y répondre, l'auteur évoque la question de la formation dans le domaine de la géographie universitaire et le contexte social, administratif et professionnel difficile dans lequel le géographe marocain évolue.

Il indique que pour répondre aux enjeux auxquels est confronté le pays, il est temps pour la géographie marocaine de se professionnaliser avec comme objectif premier de « faire profiter le territoire national d'un savoir à la fois préventif et curatif ».

Mustapha El Hannani Université d'Angers 